\title{
Regla de autoevaluación para identificar riesgo de diabetes
}

\author{
Patient self-evaluation score for diabetes risk assessment
}

Bang H y col. Ann Intern Med 2009;151:775-783.

\section{Objetivos}

Desarrollar y validar una regla de autoevaluación para detectar riesgo de diabetes y compararla con otros instrumentos ya existentes como los del Centro de Control y Prevención de Enfermedades (en inglés, CDC), la Fuerza de Tareas Preventiva de EE.UU. (en inglés, USPSTF) y el de la Asociación Americana de Diabetes (en inglés, ADA).

\section{Diseño, lugar y pacientes}

Para el desarrollo del modelo se utilizaron los datos de la Encuesta Nacional de Salud y Nutrición (en inglés, NHANES, 1999 a 2004), estudio que también participó en su validación (2005 a 2006), sumado a una cohorte combinada de dos estudios: ARIC (Atherosclerosis Risk in Communities) y CHS (Cardiovascular Health Study). Estos estudios tuvieron lugar en EE.UU. e involucraron a adultos de 20 años o más. Se utilizaron modelos de regresión logística* para determinar qué características de los participantes se asociaron independientemente con diabetes no diagnosticada.

\section{Resultados principales}

La edad, sexo, antecedentes familiares de diabetes, hipertensión, obesidad, y actividad física (tabla 1) fueron identificados y ponderados en una tabla/cuestionario (ver tabla 1). Luego se estimaron las principales características operativas para un puntaje superior a cuatro para discriminar el riesgo de diabetes (ver tabla 2).

Tabla 2: características operativas de los modelos de predicción
Tabla 1: cuestionario para evaluar prediabetes y diabetes no diagnosticada. ${ }^{*}$

\begin{tabular}{|c|c|c|c|}
\hline Pregunta & Categorias & Puntos a asignar & $\begin{array}{l}\text { Puntaje } \\
\text { parcial }\end{array}$ \\
\hline $\begin{array}{l}\text { 1. ¿Que edad tiene } \\
\text { Ud. en años? }\end{array}$ & $\begin{array}{c}<40 \\
40 \text { a } 49 \\
50 \text { a } 59 \\
\geq 60\end{array}$ & $\begin{array}{l}0 \\
1 \\
2 \\
3\end{array}$ & \\
\hline 2. ¿Es Ud. mujer o varón? & $\begin{array}{l}\text { Mujer } \\
\text { Varón }\end{array}$ & $\begin{array}{l}0 \\
1\end{array}$ & \\
\hline $\begin{array}{l}\text { 3. ¿Tiene Ud. padres } 0 \\
\text { hermanos con diabetes? }\end{array}$ & $\begin{array}{l}\text { No } \\
\text { Si }\end{array}$ & $\begin{array}{l}0 \\
1\end{array}$ & \\
\hline $\begin{array}{l}\text { 4. ¿Tiene Ud. o esta tomando } \\
\text { alguna medicación para la } \\
\text { presión arterial alta? }\end{array}$ & $\begin{array}{l}\text { No } \\
\text { Si }\end{array}$ & $\begin{array}{l}0 \\
1\end{array}$ & \\
\hline $\begin{array}{l}\text { 5. ¿Tiene Ud. sobrepeso u } \\
\text { obesidad? } \\
\text { (vea mapa más adelante } \\
\text { para contestar esta pregunta } \\
\text { con mayor precisión) }\end{array}$ & \begin{tabular}{|c|} 
No \\
Sobrepeso \\
Obesidad \\
Obesidad extrema
\end{tabular} & $\begin{array}{l}0 \\
1 \\
2 \\
3\end{array}$ & \\
\hline 6. ¿Es Ud. físicamente activo? & $\begin{array}{l}\text { No } \\
\text { Si }\end{array}$ & $\begin{array}{c}0 \\
-1\end{array}$ & \\
\hline
\end{tabular}

Si su puntaje TOTAL es igual o mayor a cuatro: Ud. presenta riesgo de diabetes 0 prediabetes no diagnosticada

Si su puntaje TOTAL es igual o mayor a cinco: Ud. está con riesgo alto para diabetes.

En ambos casos, es recomendable consultar a su médico de cabecera para que evalue la utilidad de realizar un análisis.

\begin{tabular}{l|c|c|c|c|c}
\multicolumn{1}{c|}{ Modelo } & Puntaje & \% con DBT & Sensibilidad & Especificidad & VPP* \\
\hline Derivación & $\geq 5$ & $35 \%$ & $79 \%$ & $67 \%$ & $10 \%$ \\
\hline Validación & $\geq 5$ & $40 \%$ & $72 \%$ & $62 \%$ & $10 \%$ \\
\hline Instrumentos de comparación ${ }^{\star \star}$ & & & 44 a $100 \%$ & 10 a $73 \%$ & $59 \%$ \\
\hline
\end{tabular}

\section{Conclusión}

Este cuestionario para detección de diabetes, de fácil implementación, parece demostrar mejoras con respecto a los métodos existentes. Se necesitan más estudios para evaluarla en poblaciones diversas.
*VPP: valor predictivo positivo. LR: likelihood ratio. **USPSTF, ADA. Palabras claves: Diabetes tipo 2, regla de predicción, riesgo.

Key words: type 2 diabetes, prediction rule, risk.

Fuente de financiamiento: Clinical and Translational Science Center at Weill Comell Medical College.

\section{Comentario}

La diabetes es una de las principales causas de morbimortalidad a nivel mundial y ante el aumento de su prevalencia, la prevención se ha convertido en una prioridad importante en salud ${ }^{1-4}$.

Varios estudios demuestran que los cambios de estilo de vida y algunos fármacos pueden prevenir o retrasar la diabetes y sus complicaciones, siendo intervenciones altamente efectivas para aquellas personas con mayor riesgo ${ }^{3-7}$. Existen algunos cuestionarios para identificar riesgo de diabetes con más de diez años de vigencia, los cuales demostraron no ser apropia$\operatorname{dos}^{8}$ ya que son demasiado complejos y extensos, o la gente no los completa por miedo a tener la enfermedad o requerir su tratamiento. Hasta ahora no existía una herramienta práctica para detectar aquellas personas con altas probabilidades de padecer diabetes. Sin embargo, es importante recordar que esta prueba no sirve para diagnosticar diabetes, sino que indica una probabilidad que debe ser confirmada a través de un estudio diagnóstico.

\section{Conclusiones del comentador}

Los autores lograron elaborar y validar un cuestionario simple, práctico y eficiente, que utiliza pocas preguntas, insume poco tiempo de respuesta y no requiere uso de calculadora. Naturalmente serán necesarios estudios en otras poblaciones para poder confirmar si mantiene su capacidad predictiva como se propone.

Gabriel Ruiz [ Médico Generalista. Albardón, San Juan. garuizgio@yahoo.com.ar ]

*Ver glosario

Recibido el 29/04/2010 y aceptado el 20/10/2010

Ruiz, G. Regla de autoevaluación para identificar riesgo de diabetes. Evid Act Pract Ambul 14.2 51. Abr-Jun 2011. Comentado de: Bang H, Edwards A, Andrew S, y col. Development and Validation of a Patient Self-assessment Score for Diabetes Risk. Ann Intern Med 2009;151:775-783. PMID: 19949143.

\section{Referencias}

1. Costa y col. Detección de la diabetes mellitus en consultas externas hospitalarias: utilidad de un cuestionario de cribado. Med Clin (Barc). 2003;120(8):287-91

2. Gagliardino J y col. Evaluación y costos del proceso de atención de pacientes diabéticos. Medicina (Buenos Aires) 2000;60:880-888.

3. Canadian Diabetes Association. 2008 Clinical Practice Guidelines for the Prevention and Management of Diabetes in Canada. Canadian Journal of Diabetes 2008;32 Supp 1. 3. Canadian Diabetes Association. 2008 Clinical Practice Guidelines for the Prevention and Man

4. World Health Organization. Screening for Type 2 Diabetes: Report of a World Health Organization and International Diabetes Federation meeting (en línea). Geneva 2003. Disponible 4. World Health Organization. Screening for Type 2 Diabetes: Report of a World Health Organization and In
en URL: http://www.who.int/diabetes/publications/en/screening_mnc03.pdf (ultimo acceso mayo 2011).

5. Tuomilehto J y col. Prevention of type 2 diabetes mellitus by changes in lifestyle among subjects with impaired glucose tolerance. NEJM 2001;344(18):1343-50.

6. William H y col. Predicting Risk for Diabetes: Choosing (or Building) the Right Model. Ann Intern Med. 2009;150:812-814.

7. Hoerger T. Harris R. Screening for Type 2 Diabetes Mellitus: A Cost-Effectiveness Analysis. Ann Intern Med 2004;140(9): 689-699.

8. Nijhof N y col. Determinants of the Use of a Diabetes Risk-Screening Test. J Community Health 2008;33(5):313-317. 